\title{
Les femmes dans la révolution batave : droits des femmes, droits du peuple
}

\section{Myriam Everard}

\author{
(2) OpenEdition \\ Journals \\ Édition électronique \\ URL : https://journals.openedition.org/ahrf/493 \\ DOI : 10.4000/ahrf.493 \\ ISSN : 1952-403X \\ Éditeur : \\ Armand Colin, Société des études robespierristes \\ Édition imprimée \\ Date de publication : 1 décembre 2001 \\ Pagination : 93-105 \\ ISSN : 0003-4436 \\ Référence électronique \\ Myriam Everard, «Les femmes dans la révolution batave : droits des femmes, droits du peuple », \\ Annales historiques de la Révolution française [En ligne], 326 | octobre- décembre 2001, mis en ligne le \\ 07 mars 2008, consulté le 22 avril 2022. URL : http://journals.openedition.org/ahrf/493 ; DOI : https:// \\ doi.org/10.4000/ahrf.493
}




\title{
LES FEMMES DANS LA REVOLUTION BATAVE DROITS DES FEMMES, DROITS DU PEUPLE
}

\author{
MYRIAM EVERARD
}

\begin{abstract}
Les femmes révolutionnaires bataves ont peu fait couler d'encre. Les historiens ont négligé d'enregistrer leur présence dans les fêtes ou dans les sociétés politiques. Pourtant, tout comme le peuple, les femmes ont été politisées dès la révolution des patriotes; la révolution batave leur a concédé une certaine importance, dans les cérémonies et les fêtes. Mais, comme en France, rares étaient ceux qui envisageaient de leur conférer les mêmes droits qu'aux hommes. La présente étude fait le bilan des actions féminines dans les années 1781-1798.
\end{abstract}

Mots clés : femme patriote ; République batave ; vierge de la liberté ; droits de la femme ; donatrice ; fêtes.

Le jeudi 12 avril 1792, Jan Anthony d'Averhoult, fervent patriote des années 1780 qui avait dû s'exiler en France lors de la restauration de 1787 et qui, de par son engagement dans la Révolution française, était devenu député des Ardennes à la Législative, s'adressait en ces termes à l'Assemblée :

« Messieurs, en Hollande où la Constitution française est adorée de tous les amis de la liberté, deux poètes, M. Bernard Bosc et Mlle Pétronille Moëns, une jeune fille de 20 ans, aveugle depuis sa cinquième année, ont composé des pièces en vers hollandais sur les principes de la Constitution française. Ils ont dédié leur ouvrage à l'Assemblée nationale et au roi qu'ils appellent les premiers représentants d'un peuple libre, et m'ont chargé de faire l'hommage d'un exemplaire à l'Assemblée nationale. » (1)

Avec cet hommage à la Constitution française, accueilli par des applaudissements et sanctionné par l'inscription dans les procès-verbaux d'« une mention honorable [...] pour les sentiments qui l'ont dicté et de ceux qui ont composé l'ouvrage », Bernardus Bosch et Petronella Moens avaient fait un

* Le Vertaalfonds KNAW s'est chargé des frais de traduction.

(1) Archives parlementaires de 1787 à 1860, Première série, vol. 41, Paris, 1969, p. 507.

Annales historiques de la Révolution française - $2001-N^{\circ} 4$ [93 à 105] 
acte, dont la signification politique serait perceptible jusque dans leur pays. Ce témoignage public d'attachement à la Révolution française fut signalé en effet dans la presse néerlandaise. Les auteurs publièrent aussi leurs poèmes et la dédicace ne laissait aucun doute sur leur amour de la liberté et leurs affinités avec les révolutionnaires français (2).

Petronella Moens, qui avait en fait 30 ans et était effectivement aveugle, jouissait en 1792 d'une certaine renommée en tant que poète. Ses poèmes étaient encensés par des sociétés très diverses et elle-même était membre de plusieurs sociétés littéraires, où elle était fort active sur papier et en personne (3). Elle était connue pour être une patriote convaincue et avait opté pour le parti des «braves Bataves», qui défendaient les droits des citoyens contre «l'oppresseur de la patrie », Guillaume V (4). L'éloge de la Constitution française était une suite logique de cet engagement politique. Ce qui frappe dans ce poème, c'est que, dès les premiers vers, Moens se présente comme le porte parole d'un collectif de femmes bataves :

«C'est pour vous, fils de la Liberté !

C'est pour vous que chantent les femmes néerlandaises. »

Plus loin, elle précise qui sont les femmes qu'elle représente :

«Assemblée! J'admire votre grandeur,

Ces femmes sont autant d'héroïnes guerrières

dont le courage protègera le droit. » (5)

Dans cette assurance que les femmes sont prêtes à défendre la liberté, les armes à la main, résonne l'écho de dizaines de professions de foi de ce genre, formulées par les femmes durant les années 1781-1787. Contrairement à ce qu'affirme l'historiographie traditionnelle, la période patriote connut un engagement féminin important, varié et constant. La contribution féminine y était même institutionnalisée (6). Pour mieux comprendre la participation des femmes à la vie politique de la République batave, force est donc de revenir brièvement sur leur rôle durant l'époque des patriotes.

(2) Sur l'événement, Leydse Courant, 20 avril 1792. Les poèmes ont été publiés par Petronella MOENS et Bernardus BOSCH, De nieuwe Constitutie van Frankrijk, Amsterdam, 1792.

(3) M. DE VRIES, « Dichten is zilver, zwijgen is goud. Vrouwen in letterkundige genootschappen, 1772-1800 », De Achttiende Eeuw, n 31,1999, pp. 187-213.

(4) P. MOENS, « Na net leezen van het Rapport, wegens de Actie, voorgevallen by Vreeswyk, op den 9 may, 1787, De Haagsche Correspondent, 1787, II, pp. 93-96.

(5) P. MOENS \& B. BOSCH, op. cit., p. 5 et p. 26.

(6) Ce qui suit differre sur des points importants des conclusions de R. Dekker, entre autres celles de l'article publié en collaboration avec W. TE BRAKE et L. VAN DE POL « Women and political culture in the Dutch revolutions », dans Women and politics in the age of the democratric revolution, $\mathrm{H}$. B. APPLEWHITE \& D. G. LEVY, (éd.), Ann Arbor, 1990, pp. 109-146. Ou le résumé, « Femmes et révolutions au XVIIIe siècle aux Pays-Bas», Réseaux. Revue interdisciplinaire de philosophie morale et politique, nos 61-63, 1991, pp. 22 29. Ou R. DEKKER \& J. VEGA, « Les femmes aux côtés des deux révolutions néerlandaises, de 1781-1787 et de 1794-1795 », dans Encyclopédie politique et historique des femmes. Europe, Amérique du Nord, G FAURÉ, (éd.), Paris, 1997, pp. 233-254. 


\section{Bannières, canons et héroïnes guerrières}

La révolution des patriotes (1781-1787) déclencha un grand nombre de turbulences : commentaires politiques, lettres ouvertes, dénonciations, plaintes et donna lieu à la naissance d'une presse politique dont quelques journaux étaient diffusés sur le territoire des Pays-Bas tout entier, tandis que dans chaque ville et nombre de villages étaient créées des milices patriotes qui parvinrent en quelques années à organiser un réseau national.

Dans les manifestations du mouvement qui s'accroissait et se diversifiait progressivement, dès les débuts, des femmes étaient impliquées, qui, en toute occasion, faisaient entendre leur voix. Des auteurs féminins, connus ou inconnus, des femmes écrivains, professionnelles et amateurs, apportaient des contributions au discours patriote, aux côtés de leurs collègues masculins. C'est ainsi qu'il y en eut pour chanter la bataille du Doggersbank de 1781, où le viceamiral Zoutman remporta la première victoire de la marine batave contre l'Angleterre; pour célébrer l'alliance de 1782 entre les États-Unis et la République ; pour pleurer la mort de Johan Derk van der Capellen en 1784 ; pour déplorer en 1786 l'expédition punitive de l'armée stadhouderienne contre les forteresses patriotes d'Elburg et de Hattem ou la bataille de Vreeswijk de 1787 entre les patriotes et le stadhouder (7). Quand elles mentionnent leurs contributions à la bonne cause, le style devient musclé. Motivée par son amour de la patrie, Agnita van Dyck, une femme poète peu connue, qui ne s'en aventura pas moins dans le genre patriotique, rêvait en 1783 de suivre les traces des héroïnes guerrières des légendes nationales et de prendre les armes afin de «plonger dans le sein du violeur de la paix, le britannique, ce fer acéré, avec une violence infinie » (8). Et elle regrettait que le défaut de force physique des femmes et un trop grand sentiment d'honneur masculin l'en aient empêché. En 1786, une femme ayant pour pseudonyme Androgina Plebeja, manifestait moins de retenue dans son «Adresse à Guillaume $\mathrm{V}$ », où elle menaçait celuici, au cas où il ne change point d'attitude - l'expédition contre Elburg et Hattem venait d'avoir lieu - d'aller jusqu'au bout :

« Alors cette main habituée à tenir l'aiguille, raffermie par l'acier étincelant, mènera le peuple au combat. Et si ce pays libre ne reçoit ni aide ni salut, alors je saisirai le glaive à deux tranchants pour punir le bourreau de la patrie. » (9)

(7) Quelques exemples, Anna DIERSEN, Uitspanningen in leedige uuren, gewyd aan zegepraalende helden op de Doggersbank den 5 den Augustus 1781, Amsterdam, 1782 ; Agnita VAN DYCK, Lauwerbladen voor de zonen der Vrijheid, Dordrecht, 2 vol. 1783; Adriana VAN OVERSTRATEN, Gedenkzuil voorjohan Derk van de Capellen tot den Pol, overkden te ZwoUe, den 6den van wiedemaande, desjaars 1784, Rotterdam/Haarlem, 1784, pp. 6364 ; Elisabeth BEKKER, veuve WOLFF, Vrijheid, blijheid Vaderlandsch dichtstuk, 's Gravenhage, 1786.

(8) A. VAN DYCK, « De trouw voor het Vaderland », Lauwerbladen voor de zonen der Vrijheid, vol. 2, Dordrecht, 1783, p. 66.

(9) Androgina PLEBEJA, Aanspraak van een Vaderlandsche vrouwe aan Willem de Vde, Deventer, 1786. 
Mais plus importante encore est la contribution des femmes dans les organisations paramilitaires et politiques qui constituent le cœur du mouvement patriote : la milice. Bien que ces sociétés privées aient été encouragées par certaines administrations municipales, ce sont elles, en principe, qui devaient assumer leurs dépenses en matière d'uniforme, d'armement, d'hébergement, d'entraînement. Certes, il y avait aussi des membres donateurs, qui ne participaient pas à l'entraînement mais contribuaient au paiement des frais, et, surtout, il y avait des donatrices. Dans ses mémoires, Pieter Vreede, rappelle qu'elles étaient fort nombreuses : «il y en avait bien des milliers » grâce aux donations desquelles les milices purent fonctionner (10). Mais parmi ces donatrices, il en est qui font plus que payer une contribution annuelle. Certaines prêtent leurs champs pour l'entraînement, d'autres distribuent des offrandes, parmi lesquelles prime la bannière : dans des dizaines de villes et de villages, les femmes, pour la plupart issues de familles patriotes renommées, faisaient faire des bannières et les offraient à leur milice. Cette offrande se faisait selon un cérémonial emprunté à la tradition, qui voulait que le stadhouder ou son représentant donne une bannière aux milices municipales. Cette cérémonie symbolisait que les milices étaient liées à la patrie. Les patriotes reprennent le cérémonial mais substituent au stadhouder les femmes et ce sont à elles désormais que les milices adressent leur serment de fidélité à la patrie. Dans les discours tenus en ces occasions, les femmes adoptent le ton de la situation : elles ordonnent aux milices de défendre le droit et la liberté et de mourir plutôt que d'abandonner leur étendard. Quelques-unes sont plus belliqueuses et assurent aux milices qu'elles aussi sont prêtes à prendre les armes et à tout quitter pour les rejoindre et sauver la patrie.

La reconnaissance des milices envers ces manifestations féminines de soutien n'était pas négligeable. En témoignent les remerciements qui ponctuaient les cérémonies, où les hommes confirmaient leur volonté de bien remplir leur tâche et de défendre leur étendard jusqu'à leur dernière goutte de sang. Ces remerciements, de même que les discours des donatrices, étaient presque toujours publiés, de sorte que les donatrices n'étaient pas seulement vénérées par la milice du cru, mais aussi par la patrie tout entière. De temps à autre, les milices manifestaient leur reconnaissance d'une autre façon. C'est ainsi que la milice de Groningue remercia la donatrice Sara Verdion, douairière Van Maneil, qui lui avait donné en 1786 une somme importante et trois canons, en organisant un vaste défilé devant sa maison et en tirant une salve en son honneur, et, quand elle décéda début 1787, la compagnie l'enterra avec tous les honneurs militaires (11).

(10) P. VREEDE, Mijn levensloop, Hilversum, 1994, p. 52. 3 avril 1787.

(11) Nieuwe Nederlandsche Jaerboeken, 1786, pp. 746-747. Groninger Courant, 13 octobre 1786 et 
Ces cérémonies publiques, qui étaient entourées de toute la publicité possible et imaginable, contribuaient à la réputation patriote des donatrices bien au-delà de leur communauté. Si dans leur propre milieu elles croulaient sous les honneurs, dans le camp orangiste, elles étaient accablées de sarcasmes et d'insultes : les caricatures les ridiculisaient sous l'image de «viragos armées » ou sous celle de «filles de troupe ». Dans la presse orangiste, elles étaient insultées et désignées par leurs nom et prénom. Et lorsque les hostilités entre patriotes et stadhouderiens débouchaient sur de véritables combats, les donatrices, tout comme les patriotes, étaient une cible privilégiée. Une des premières à en subir les effets fut la donatrice de Hattem, Anna Geertruida Tulleken : lors de l'expédition punitive de l'armée orangiste contre les villes d'Elburg et de Hattem, sa maison fut pillée et saccagée (12). Et après qu'en septembre 1787, l'armée prussienne était venue à l'aide de Guillaume $\mathrm{V}$ et, en quelques jours, avait battu les patriotes, nombre de donatrices furent victimes des velléités vengeresses des orangistes. Parmi elles, Bartha van Crimpen, épouse d'Ary van der Meer, membre honoraire de la milice haguenoise, dont la maison fut totalement pillée et dévastée. Aussi nombre d'entre elles, dont Bartha van Crimpen, s'enfuirent-elles en France où elles restèrent des années en exil, en compagnie de leurs proches masculins qui, eux aussi, bien souvent, avaient lutté pour la cause patriote (13).

Avec la restauration de 1787, les milices patriotes furent supprimées; leurs armes confisquées et leurs bannières déchirées, de préférence sur la place publique; les fonctionnaires patriotes furent remplacés par des orangistes; nombre de patriotes condamnés par contumace. Le stadhouder fut restauré dans ses droits et, pendant quelques années, le parti orangiste triompha. En quelques semaines, se calmèrent les brutalités à l'endroit de quiconque passait pour patriote, même si dans les années suivantes l'on constate encore des voies de fait contre des patriotes, qui n'épargnaient pas les femmes. En 1790 - et le 14 juillet, de surcroît - deux Amstellodamoises, soupçonnées d'avoir des sympathies patriotes, furent ainsi jetées à l'eau (14).

Il n'en demeure pas moins, surtout après 1789, que diverses publications voient le jour, où sont chantés les idéaux patriotiques et où, l'œil fixé sur la France, est exprimé l'espoir d'une révolution patriote en terre batave. Ici aussi des voix de femmes se font entendre. Notamment celle de

(12) Informatien wegens de plunderingen en de geweldenarijen van de militie te Hattem, op verzoek van den Raadvan Staaten, ingewonnen doorden magistraat der stad Zwolle, Utrecht, 1786, pp. 13-14.

(13) M. EVERARD, «Vrouwen voor het vaderland : burgeres Van der Meer en de Bataafse politiek », Holland, n'31, 1999, pp. 272-281. Que les 2000 femmes ayant fui en 1787 n'aient fait que suivre leurs époux ou amis et n'aient pas eu à pâtir d'une «répression de la part de la populace orangiste », ainsi que l'affirme J. ROSENDAAL, est donc une interprétation incorrecte. «Parce que j'aime la liberté, je retourne en France », dans Le Voyage révolutionnaire, (éd. W. FRUHOFF \& R. DEKKER), Hilversum, 1991, p. 43.

(14) N. D'AUBIGNY, xNiet zo erg Hollands». Dagboek van een reis naar Nederland (1790-1791), Hilversum, 2001, p. 46. 
Petronella Moens. Non seulement elle publie son éloge à la Constitution française, mais, en 1792, elle sort également un recueil de Liederen voor het Vaderland ( Chants pour la Patrie »), écrit en collaboration avec Bernardus Bosch dont quelques poèmes sont dédiés aux patriotes exilés; en 1793, à l'occasion de la mort de Zoutman, elle donne une contribution dans un recueil, où le vice-amiral est célébré comme un véritable héros de la patrie ; en 1794, elle se réjouit de l'abolition de l'esclavage, décrétée par la Convention, et la salue comme un des bienfaits de la Révolution française (15). Que les femmes s'attendent à ce que ces bienfaits leur reviennent en partie et qu'elles soient délivrées de la tyrannie masculine, c'est là un espoir explicitement formulé en 1794 dans les milieux patriotes et une idée entretenue sous la forme d'un refrain : dans un nouveau chant sur l'air d'une mélodie consacrée à Zoutman, une «femme de tête» y chante la «véritable égalité » qui devra s'étendre jusque dans le mariage :

«Oj'espère, de tout mon cœur,

que la philanthropique France

modifiera un jour notre sort ;

C'est le devoir d'un patriote

de libérer aussi les femmes. » (16)

À la même époque, dans plusieurs villes, les patriotes se regroupent dans des comités révolutionnaires clandestins. Ils entrent en contact avec le Comité révolutionnaire batave, créé en 1792 par les patriotes exilés en France, afin de préparer une révolution dans leur pays. Fin juillet 1794, lors d'une assemblée nocturne qui se tient dans une auberge à la périphérie de Haarlem, les comités locaux décident de s'unir en une organisation nationale (17). La réunion avait pu avoir lieu parce que la propriétaire, Christina Koets, avait mis son auberge à la disposition des patriotes. Si tant est que son rôle fut de second plan dans cette affaire, par ce geste, elle n'en courait pas moins les mêmes risques que les autres « conjurés ».

\section{Les Vierges de la Liberté et les clubs de femmes}

L'idée d'une révolution en Hollande devient réalité à l'automne 1794, quand l'armée française, accompagnée d'une légion d'exilés patriotes, ellemême commandée par le patriote de Hattem, Herman Willem Daendels,

(15) P. MOENS \& B. BOSCH, Liederen voor het Vaderland, Dordrecht \& Bergen op Zoom, 1792. $\mathrm{R}$ MOENS, Ter nagedagtenis van wijlen zijne excellentie den heer Johan Arnold Zoutman, vice-admiraal van Holland en Westfriesland Gouda, 1793, pp. 52-56 et idem, Vruchten der eenzaamheid, Amsterdam, 1798, pp. 24-27.

(16) «De denkende vrouw », Bundel van Bataafsche volks-liedjens, op de meest bekende en nieuwste opera-wijzen, Zaandam, s.d. (1794), p. 43.

(17) T. JORISSEN, Depatriotten te Amsterdam in 1794, Amsterdam, 1875, p. 64. 
conquiert la province méridionale du Brabant. Au nord des fleuves, les comités révolutionnaires avertissent les sociétés de lecture et les clubs populaires qui forment leur arrière-ban de se tenir prêts. Aussi, après que les troupes franco-bataves aient traversé les fleuves et atteint les provinces septentrionales; après que Guillaume V se soit embarqué le 18 janvier 1795 pour l'Angleterre et avant même que n'arrivent les troupes françaises, éclatent ici et là des révolutions locales. À Haarlem, où, dès le 19 janvier, le comité révolutionnaire local a déposé la municipalité en place, la révolution a été fort bien préparée. Le même jour est érigé un arbre de la Liberté, et, il s'avère que, dans ces préparatifs, des femmes étaient impliquées :

«Saluées par des cris de joie, qui fusaient de tous côtés, quelques femmes arrivèrent, qui s'étaient constituées en club. Elles portaient un sapin, qui devait symboliser l'arbre de la Liberté ; à leur tête, se trouvait C. Koets, aubergiste de « l'Arme d'Amsterdam. » (18)

Le club de femmes, avec à sa tête, Christina Koets, qui, comme on vient de le voir, avait abrité chez elle les comités révolutionnaires dans l'été 1794, n'était pas une création de circonstance. Dans la description officielle des événements du 19 janvier 1795, que publiera le comité révolutionnaire quelques jours plus tard, le club est décrit comme réunissant quelques « femmes éprises de liberté, qui animées du plus pur zèle pour la Liberté et la Patrie, ont constitué un club depuis quelque temps ». Ce club de femmes a été admis dans la galerie d'honneur, publiée par le comité, réunissant une soixantaine de clubs populaires grâce aux efforts desquels la révolution avait pu s'effectuer. Que les femmes aient eu une place d'honneur dans les festivités autour de l'arbre de la liberté, n'est pas un hasard : ce sont elles qui avaient offert l'arbre à la municipalité, après l'avoir fait décorer à leurs frais. Une initiative qui leur valut des remerciements officiels et publics de la part de la municipalité provisoire. Ces remerciements révèlent également que le club de femmes avait joué un rôle important dans les fêtes qui avaient accueilli les troupes françaises le 20 janvier 1795, tandis qu'elles s'étaient occupées par ailleurs de la collecte de vêtements et de literie, à l'usage des Français (19).

Pour ce qui est des autres villes, les organisations et les activités féminines dans les préparatifs révolutionnaires ne sont pas connues. Certes, dans la nouvelle République batave, des clubs de femmes virent le jour ailleurs, qui s'adaptèrent aux exigences des temps nouveaux et se chargèrent entre autres de la décoration des arbres de la Liberté ou bien de trouver les

(18) C. DE KONING, Tafereel der stad Haarlem en derzelver geschiedenis van de vroegste tijden aftot op den tegenwoordigen toe, vol. 3, Haarlem, 1808, p. 304.

(19) Kort verhaal van de revolutie in Haarlem, op den 19 January 1795, Haarlem, 1795, p. 16 et pp. 55-56. De commissie der provisioneele stads-regeering binnen Haarlem aan die burgeressen welke zich hebben uitgezonderd door het schenken van den vryheids-boom, s.l., s.d. (Haarlem, 1795). Nieuwe Nederlandsche Jaerboeken, 1795, p. 777. 
bandages et les charpies nécessaires pour les soldats français blessés. Il y avait aussi des femmes qui, suivant la tradition patriote, apportaient une contribution personnelle. Ce pouvait être un arbre, de l'argent et des bijoux, ou bien, une bannière qui n'avait pas été terminée à temps en 1787 pour être donnée à la milice locale. Et, comme à l'époque des patriotes, quand il y avait un événement d'envergure nationale, les femmes ne demeuraient pas en reste pour exprimer publiquement leurs sentiments patriotiques ; elles manifestaient leur engagement politique dans des cantates, des poèmes commémoratifs et des chants dédiés à «l'heureuse révolution ». Parmi elles, des auteurs renommés, comme Petronella Moens, mais aussi des femmes qui se manifestaient pour la première fois en public, telle Maria Roelofswaert, sœur d'un éditeur patriote de Delft (20).

Entre toutes les nouveautés introduites par la révolution batave, force est de mentionner les grandes fêtes de la Liberté, organisées dès les débuts de la République batave. Ce qui était inédit notamment, était l'ampleur de la mobilisation des femmes et la place significative qui leur fut conférée dans les célébrations de l'ordre nouveau. Si autrefois les femmes en tant que donatrices avaient été présentes dans les réunions publiques des milices patriotes locales et si elles y avaient de temps à autre pris la parole, dans les centaines de fêtes célébrées au cours des premiers mois de 1795, non seulement la présence féminine s'est amplifiée, mais, dans les festivités, elles se voient confier un rôle actif, en tant que «filles de la Liberté», «femmes de la Patrie » ou «Citoyennes ». Dans les cortèges festifs, représentant les idéaux de la Révolution, les femmes ont des fonctions diverses et variées. Revêtues d'un costume uniforme dans presque toutes les fêtes - robes blanches toutes simples, ornées d'un ruban tricolore - elles figurent les idéaux égalitaires de la révolution, qui ne tolèrent plus les distinctions aristocratiques. Parfois, elles font partie d'un groupe de jeunes orphelines, de religions différentes, qui marchent derrière une bannière de la Liberté; elles incarnent ainsi la liberté religieuse et le fait que l'ordre politique nouveau ne veut plus d'Église officielle. D'autres fois, elles portent le drapeau national ou des boucliers ornés d'allégories, telles que les Droits de l'homme et du citoyen. Dans certaines fêtes marche en tête du cortège une femme, tenant une lance, surmontée du chapeau de la Liberté ; dans d'autres, des jeunes filles portent une cage et, au moment suprême, libèrent les pigeons qui y étaient enfermés ; et puis, il y a des femmes qui accueillent avec des mots de bienvenue les patriotes de retour ou les membres des nouvelles municipalités (21).

(20) Zege-feest der burger-schuttery te Oostzaandam op het Kalf, by net ontvangen van het vaandel, aan haar vereerd door de burgeresse Neeltje de Vries, uit naam haarer grootmoeder de burgeresse Guurtje Jut, huisvrouw van den burger Klaas Taan, dejonge. Op zondagden 12 julij 1795, Zaandam, s.d. (1795). P. MOENS, De zegevierende patriotten in Nederland. Cantate, Amsterdam, 1795. M. ROELOFSWAERT, Triumphzang aan Nederland, ter gelegenheid van de gelukkige omwenteling in January 1795, s.l., s.d. (Delft, 1795).

(21) Sur le rôle des femmes dans les fêtes, M. EVERARD \& M. AERTS, «De burgeres. Geschiedenis van een politiek begrip », à paraitre dans J.J. KLOEK \& K. TILMANS (éd.), Burger (vol. 4 de l'histoire néer- 
Avec le temps, les représentations révolutionnaires et les cortèges des fêtes deviennent plus riches et plus théâtraux. Lors des fêtes de l'alliance de juin 1795, organisées en l'honneur du traité avec la France, ici et là, des feux brûlent les symboles aristocratiques (armes et perruques); des tableaux vivants représentent des esclaves libérés de leurs chaînes ou des patriotes rapatriés embrassés par leur mère. Et puis, outre les jeunes filles libérant les pigeons ou les femmes arborant la lance et le chapeau de la Liberté, on trouve dans les cortèges des Vierges de la Liberté revêtues de tous leurs atours. Un des points forts de ces fêtes est celui du 3 mars 1796 à La Haye, où fut célébrée l'ouverture de l'Assemblée nationale. Au centre du cortège qui traversa des heures durant les divers quartiers de la ville et où se trouvaient non seulement des tableaux vivants, des citoyens en armes, des soldats français, des membres de la municipalité et des sociétés fraternelles, mais encore des femmes et des jeunes filles revêtues de blanc, dominait, debout dans un char triomphal, la Liberté, personnifiée par une femme vêtue à l'antique, armée d'une lance et du chapeau de la Liberté. Elle symbolisait dans le même temps l'idée clé de la fête : la liberté du Peuple batave qui pour la première fois avait élu lui-même son gouvernement.

Que la Liberté ait été figurée par une femme, est sans nul doute dicté par la tradition iconographique. Mais que les organisateurs, si soucieux de composer le cortège avec soin et de faire en sorte qu'il y ait un équilibre entre hommes et femmes et entre les diverses religions, aient choisi de faire figurer une femme en chair et en os, indique un choix conscient, d'autant plus qu'ils n'ont pas sélectionné une personne quelconque ou une beauté locale, mais la «citoyenne »Van der Meer, la donatrice haguenoise, évoquée plus haut, qui venait de rentrer de son long exil en France. La signification de la présence ostentatoire d'une (de cette) femme, en cette qualité et en cette circonstance, englobe plusieurs niveaux. Manifestement, de par son passé politique, Bartha van der Meer fonctionne comme la représentante des patriotes qui ont souffert et se sont battus pour la patrie; dans sa personne, sont comprises évidemment toutes les femmes; et, en elle, enfin, sont honorés tous les patriotes, hommes et femmes (22). La fête de l'alliance était donc aussi une fête des patriotes, amplement représentés -parmi les milices, les membres de la municipalité et les sociétés fraternelles, qui suivaient le char de la Liberté. Pourtant, la fête ne se trouvait pas seulement sous le signe du passé : c'était aussi et surtout une fête du Peuple batave, qui, avec les élections des députés de l'Assemblée nationale, venait de recouvrer sa liberté et de faire acte de souveraineté. À première vue, la

landaise des concepts). Voir aussi F. GRUZENHOUT, Feesten voorhet Vaderiand. Patriotse en Bataafse feesten 1780-1806, Zwolle, 1989. Grijzenhout n'attribue aucune signification spécifique à la présence des femmes dans les fêtes bataves.

(22) Beschryving der plegtigheden by het Burgerfeest in Den Haag op den 3 maart 1796, het tweede jaar der Bataafsche Vryheid, Den Haag, 1796, p. 4. 
présence de Bartha van der Meer dans une telle fête pourrait donc sembler déplacée, même si elle représente la Liberté souveraine sous la figure explicite de citoyenne. Car les femmes n'avaient pas eu le droit de vote lors des élections des représentants de l'Assemblée, tandis que l'exclusion des femmes du suffrage avait été à peine contestée. Certes, au cours des délibérations de 1795 sur la loi qui devait déterminer comment et par qui l'Assemblée nationale serait élue, était paru un texte (anonyme), où l'auteur plaidait pourtant en faveur d'une entière égalité des droits entre hommes et femmes, de sorte que les femmes puissent voter et être élues. Mais il n'avait provoqué aucun vaste débat public et les articles du projet constitutionnel évoquaient seulement les citoyens, sans mentionner les femmes, pas même dans les catégories exclues du suffrage (23). À y regarder de plus près, la présence prééminente de Bartha van der Meer a cependant une signification en relation avec l'actualité et le fait qu'elle soit qualifiée de « citoyenne » n'est pas vide de sens. La clé réside dans la participation à la fête d'un éminent défenseur des droits civiques des juifs, Mozes Cohen Belinfante, qui, bien que n'ayant pas non plus pu participer aux élections de l'Assemblée nationale, faisait partie du cortège en tant que «citoyen ». Sa présence n'est pas difficile à comprendre : il était secrétaire des sociétés fraternelles de La Haye et en tant que tel, un des principaux organisateurs de la fête. Depuis 1795, il était membre actif de la milice haguenoise. Bien que privé de droits civiques, il s'était déjà manifesté en tant que « citoyen », comme s'il anticipait pour ainsi dire sur une citoyenneté qu'il acquerrait quelques mois plus tard. C'est cette « citoyenneté par anticipation » qu'exprime, mais aussi sanctionne la présence de la citoyenne Van der Meer. Et, quand on reconsidère les activités politiques des femmes dans la République batave, elle en annonce également l'accomplissement (24).

La participation active des femmes dans les fêtes des premiers mois de la révolution n'est qu'une des facettes où elles s'affichent comme une partie du peuple au nom duquel a été proclamée la République batave. Dès les premiers moments, des femmes ont en effet fait usage de leur droit de pétition et se sont adressées au nouveau gouvernement. En janvier 1795, la citoyenne Maria Muller van den Bos, née Van den Broek, alias Mietje Bos, par exemple, demandait au Comité révolutionnaire d'Amsterdam la permission d'écrire et d'éditer un journal. On lui répondit qu'étant donné la liberté de la presse, chacun était libre d'agir dans ce sens. En septembre 1795, les couturières de Bois-le-Duc envoyèrent une pétition, dans laquelle elles

(23) P. B.v.W., Ten betooge dat de vrouwen behooren deet te hebben aan de regeering van het land, Harlingen, 1795. G.W. B ANNIER, Grondwetten van Nederland, Zwolle, 1936, pp. 17-35.

(24) Sur les activités politiques de Belinfante, J. DIVENDAL, « Mozes Cohen Belinfante. Jew to the depth of his soul», Studia Rosenthaliana, n³1, 1997, pp. 94-138. Au sujet de l'exclusion politique des femmes et de leurs pratiques politiques sur l'allégorie vivante, cf. M. AGULHON, Marianne au combat. L'imagerie et la symbolique républicaines de 1789 à 1880, Paris, 1979; D. GODINEAU, «Femmes en citoyenneté : pratiques et politiques », A.H.R.F, 1995, pp. 197-207. 
priaient la municipalité d'intervenir contre le monopole exercé par la corporation des tailleurs qui privilégiait ses membres et opprimait les couturières. Leur revendication en faveur de l'égalité se réfère explicitement aux «droits reconnus de l'homme et du citoyen» et elles se permettent même de commencer leur lettre par un jeu de mots : «Liberté, Égalité ou Combat d'aiguilles » (25).

Début 1796, Petronella Moens voit ses possibilités accrues de participer au débat politique de façon autonome. Bernardus Bosch est élu membre de l'Assemblée nationale et Moens reste seule pour diriger le journal $D e$ Menschenvriend («Le Philanthrope »), dont elle assumait depuis 1793 la rédaction avec Bosch. Au cours de la semaine où la citoyenne Van der Meer traverse La Haye, sous les traits de vierge de la Liberté, un numéro du journal est publié, où Moens plaide en faveur du droit de vote des femmes dans les assemblées primaires (26). L'Assemblée nationale n'accordera pas ce droit. Les activités des femmes ne s'en accroissent pas moins, où elles se donnent pour des membres à part entière de la communauté politique, tandis que celle-ci semble les accepter en tant que tels. Petronella Moens devient ainsi membre de mérite de la Société en faveur de la véritable religion, de la vertu, des arts et des sciences, ce qui lui donne un statut égal à celui de nombre de patriotes éminents : héros d'antan, nouveaux représentants, membres de la nation juive, mais aussi anabaptistes, catholiques, francs-maçons, libraires, savants et hommes de sciences, et, une société choisie de théophilanthropes français. Pour mieux comprendre ce qu'était cette société, qui, d'après la liste de ses membres, semble attachée à la tolérance religieuse et, du point de vue politique, se situe du côté radical et démocrate, il est utile de consulter ses Werken (« Euvres »), un des seuls périodiques néerlandais à accorder un article à la traduction néerlandaise de Vindication of the rights of woman de Mary Wollstonecraft et, ce qui est encore plus surprenant, à recommander la lecture de ce «manuel pour toutes les femmes » (27).

En 1797, alors que l'Assemblée nationale consulte le peuple sur son projet de Constitution, les activités politiques des femmes s'intensifient. Qu'elles n'aient pas le droit de participer au plébiscite, ne les empêche nullement de s'exprimer à ce sujet. Alors que De Menschenvriend publie les « pensées libres d'une citoyenne néerlandaise sur la constitution », ailleurs, dans les milieux radicaux des clubs populaires amstellodamois, des femmes

(25) M. EVERARD, «In en om de (Nieuwe) Bataafsche Vrouwe Courant. Het aandeel van vrouwen in een revolutionaire politieke cultuur », Mededelingen van de Stichting Jacob Campo Weyerman, $\mathrm{n}^{\circ} 24,2001$, pp. 67-87. B. PANHUYSEN, Maatwerk, Kleermakers, naaisters, oudkleerkopers en de gilden (1500-1800), Amsterdam, 2000, p. 222.

(26) De Menschenvriend, 1796, pp. 67-69.

(27) Werken van het genootschap, ter bevordering van waaren godsdienst, deugd, kunst en wetenschap, opgericht in de Bataafsche Republiek, Grave, 1797, p. 58. Voir aussi H. B. GRÉGOIRE, Histoire des sectes religieuses, Paris, 1810, vol. 2, pp. 79-81. Grégoire y évoque « la poète aveugle, Mademoiselle Pétronille Moens ». 
s'impliquent directement dans l'actualité politique et tentent d'en influencer le cours. Deux d'entre elles se trouvent au premier plan : Johanna van Haren, née Beaumont, et Catharina Heybeek, tandis qu'à l'arrière-plan, Mietje Bos, auteur et éditeur de YOprechte Nationale et Bataafsche Courant, à partir de 1797 , leur procure pour un temps un support où s'exprimer. La spécialité de Van Haren devient le commentaire politique en vers, dont dès les premiers mois de 1797 elle pourvoit régulièrement les journaux des clubs populaires. Elle essaie ainsi d'exercer une influence, entre autres lorsque le projet de Constitution, jugé «aristocratique» par les clubs populaires, est déposé dans les assemblées primaires. Consciente qu'elle fait partie du «sexe, exclu du droit de suffrage », elle appelle les citoyens à rejeter l'infâme projet, à maudire la tyrannie et à protéger leurs droits pour conserver leur liberté (28).

Heybeek, qui entre en scène au même moment, va plus loin encore : dans la campagne contre le projet, durant laquelle elle a fourni des contributions journalistiques à foison, elle appelle ses lecteurs à chasser par la force des assemblées primaires les partisans du projet. Cette incitation à la violence lui vaut d'être arrêtée par la suite et condamnée à trois ans de prison. Mais, entre-temps, le projet a été rejeté.

Fin 1797, alors que les discussions sur le nouveau projet de constitution débouchent sur une impasse, parce que la minorité radicale de l'Assemblée nationale n'est pas prête à faire des concessions, un mouvement national issu des clubs populaires appuie les vues radicales, en faveur d'une influence populaire, garantie par la constitution. Au même moment, Van Haren rédige également une adresse pour l'Assemblée nationale et recueille en peu de temps une centaine de signatures de femmes - initiative appuyée par Bos et son journal, que, de sa prison, Heybeek essaie aussi de favoriser en appelant toutes les femmes à faire entendre leur voix et à signer l'adresse. Le succès rapide de cette initiative suggère l'existence d'un réseau féminin, voire d'un club féminin, facilement accessible, et, donc, une capacité d'organisation, dont jusqu'à présent on ignorait l'existence. L'adresse révèle en outre la motivation profonde de cette activité politique féminine. C'est en effet en tant que «Femmes bataves, qui considèrent comme étant leur droit le droit du peuple tout entier » qu'elles présentent leurs revendications aux démocrates radicaux de l'Assemblée nationale et qu'elles les incitent à ne pas bafouer les principes de Liberté, d'Égalité et de Souveraineté populaire. En tant que femmes du peuple batave, elles revendiquent donc le droit « de changer, d'améliorer en tout temps [...] la constitution, ou bien d'en concevoir une nouvelle» - ainsi qu'il est proclamé dans les Droits de l'homme et

(28) De Menschenvriend, 1797, pp. 345-354. Sur les trois activistes des clubs populaires, M. EVERARD, «In en om de (Nieuwe) Bataafsche Courant, op. cit.. J. J. VAN HAREN, née BEAUMONT, Extra Nationaale Bataafsche Courant van Lieve van Ollefen, 7 août 1797. 
du citoyen. L'idée d'une citoyenneté à part entière n'est pas loin : quand, le 22 janvier 1798, les représentants, au profit desquels les femmes ont exercé leurs droits, prennent le pouvoir à la suite d'un coup d'État, afin de mener à bien la réalisation d'une constitution radicale, Van Haren y voit le signe qu'elle et ses collègues ont été écoutées : «Réjouissez-vous Bataves ! La fin de l'oppression approche! Réjouissez-vous, mes sœurs! Votre voix sera entendue à l'Assemblée nationale ! » (29)

Le gouvernement radical n'a pourtant pas fait grand-chose pour les femmes : Heybeek demeura en prison et la Constitution, acceptée en avril 1798, n'accordait le droit de suffrage qu'aux hommes. Mais les femmes ne renoncèrent pas pour autant aux activités politiques : Van Haren continua à faire des commentaires politiques en vers et Bos à publier son journal; Moens lança en mai 1798 un nouveau périodique, De Vriendin van't Vaderland («L'Amie de la Patrie»). On ne saura jamais si ces activités auraient pu mener à une plus grande participation politique des femmes, car, le 12 juin 1798, les radicaux furent évincés du pouvoir par un coup d'État des modérés. Peu après, Van Haren se fit silencieuse ; Bos, qui avait mis son journal au service des hommes du 12 juin, se retira de l'arène politique et, Moens, en des termes qui en disent long, se distancia de « l'état catastrophique de Révolution, qui a ébranlé depuis trop longtemps notre patrie et l'a menée quasiment à sa perte ». En véritable amie de la Patrie, elle lançait un message de réconciliation et de fraternisation d'où était absente toute revendication en faveur de l'égalité des droits des citoyennes. Avec les Constitutions de 1801 et de 1805 et surtout avec l'instauration du Royaume de Hollande en 1806, disparaîtront de la scène publique les citoyens. Il faudra attendre soixante-quinze ans avant qu'ils n'y reviennent, avec, à leurs côtés, les citoyennes (30).

(traduction du néerlandais : A. Jourdan)

Myriam EVERARD

Plantage 6

N. L. - 2311 JC Leiden

(29) «Request van onderscheiden Vaderlandsche Vrouwen» (29 décembre 1797), dans Dagverhaal der handelingen van de Nationale Vergadering representeerende het volk van Nederland, vol. 8, Den Haag, 1797, p. 255. G. W. B ANNIER, op. cit., p. 17. VAN HAREN, Oprechte Nationaale Courant, 25 janvier 1798.

(30) De Vriendin van't Vaderland, [1798], p. 162. Sur la conclusion, voir aussi, M. EVERARD \& M. AERTS, op. cit. 